OPEN ACCESS

Edited by:

Antonio Greco,

University of Catania, Italy

Reviewed by:

Felice Gragnano,

University of Campania Luigi

Vanvitelli, Italy

Marco Di Maio,

University of Salerno, Italy

Claudio Laudani,

University of Catania, Italy

Marco Spagnolo,

Universit'a degli Studi di Catania, Italy

*Correspondence:

Shaoping Nie

spnie@ccmu.edu.cn

Yaling Han

hanyaling@263.net

†These authors have contributed equally to this work

Specialty section:

This article was submitted to

Coronary Artery Disease,

a section of the journal

Frontiers in Cardiovascular Medicine

Received: 01 September 2021 Accepted: 11 November 2021

Published: 13 December 2021

Citation:

Wang X, Zhao G, Zhou M, Ma C, Ge J, Huo Y, Smith SC Jr, Fonarow GC,

Hao Y, Liu J, Morgan L, Gong W, Yan Y, Liu J, Zhao D, Han Y and Nie S (2021) Trends in Bleeding Events Among Patients With Acute Coronary Syndrome in China, 2015 to 2019: Insights From the CCC-ACS Project.

Front. Cardiovasc. Med. 8:769165. doi: 10.3389/fcvm.2021.769165

\section{Trends in Bleeding Events Among Patients With Acute Coronary Syndrome in China, 2015 to 2019: Insights From the CCC-ACS Project}

\begin{abstract}
Xiao Wang ${ }^{1 \dagger}$, Guanqi Zhao ${ }^{1 \dagger}$, Mengge Zhou ${ }^{2}$, Changsheng $\mathrm{Ma}^{3}$, Junbo $\mathrm{Ge}^{4}$, Yong Huo ${ }^{5}$, Sidney C. Smith Jr. ${ }^{6}$, Gregg C. Fonarow ${ }^{7}$, Yongchen $\mathrm{Hao}^{2}$, Jun Liu ${ }^{2}$, Louise Morgan ${ }^{8}$, Wei Gong ${ }^{1}$, Yan Yan ${ }^{1}$, Jing Liu ${ }^{2}$, Dong Zhao ${ }^{2}$, Yaling Han ${ }^{9 *}$ and Shaoping Nie ${ }^{1 *}$ on behalf of the CCC-ACS Investigators
\end{abstract}

\begin{abstract}
${ }^{1}$ Center for Coronary Artery Disease, Beijing Anzhen Hospital, Capital Medical University, Beijing, China, ${ }^{2}$ Department of Epidemiology, Beijing Anzhen Hospital, Capital Medical University, Beijing Institute of Heart, Lung and Blood Vessel Diseases, Beijing, China, ${ }^{3}$ Department of Cardiology, Beijing Anzhen Hospital, Capital Medical University, Beijing, China, ${ }^{4}$ Zhongshan Hospital, Fudan University, Shanghai, China, ${ }^{5}$ Department of Cardiology, Peking University First Hospital, Beijing, China, ${ }^{6}$ Division of Cardiology, University of North Carolina, Chapel Hill, NC, United States, ${ }^{7}$ Divisions of Cardiology, University of California, Los Angeles, Los Angeles, CA, United States, ${ }^{8}$ International Quality Improvement Department, American Heart Association, New York, NY, United States, ${ }^{9}$ Cardiovascular Research Institute and Department of Cardiology, General Hospital of Northern Theater Command, Shenyang, China
\end{abstract}

Objective: Major bleeding is a common complication following treatment for an acute coronary syndrome (ACS) and is associated with increased mortality. We aimed to explore the temporal trend of bleeding events in relation to changes of therapeutic strategies among patients hospitalized for ACS in China.

Methods: The CCC-ACS project (Improving Care for Cardiovascular Disease in China-Acute Coronary Syndrome) is a collaborative initiative of the American Heart Association and the Chinese Society of Cardiology. We analyzed 113,567 ACS patients from 241 hospitals in China from 2015 to 2019. Major bleeding was defined as intracranial bleeding, retroperitoneal bleeding, a decline in hemoglobin levels $\geq 3 \mathrm{~g} / \mathrm{dL}$, transfusion with overt bleeding, bleeding requiring surgical intervention, and fatal bleeding. Kruskal-Wallis test was used to examine the trend of major bleeding over time.

Results: The rate of in-hospital major bleeding decreased from $6.3 \%$ in 2015 to $4.7 \%$ in 2019 (unadjusted $\mathrm{OR}=0.74,95 \% \mathrm{Cl}$ : 0.68-0.80, and $P<0.001$ ). The relative changes were consistent across almost all subgroups including patients with NSTE-ACS and STEMI, although the trend was more pronounced in NSTE-ACS patients. The decrease in bleeding was accompanied by a decrease in use of GP IIb/llla inhibitors and parenteral anticoagulation therapy during hospitalization. The annual reduced risk of bleeding (OR $=0.91,95 \% \mathrm{Cl}$ : 0.89-0.93) was attenuated after stepwise adjusting for baseline characteristics and antithrombotic treatments ( $\mathrm{OR}=0.95,95 \% \mathrm{Cl}: 0.93-0.97$ ), but did not change after adjusting for invasive treatment ( $\mathrm{OR}=0.95,95 \% \mathrm{Cl}: 0.93-0.97$ ).

Conclusions: There was a temporal reduction in in-hospital bleeding among Chinese ACS patients during the last 5 years, which was associated with more evidence-based use of antithrombotic therapies. 


\section{Clinical Trial Registration: https://www.clinicaltrials.gov, identifier: NCT02306616.}

Keywords: acute coronary syndrome, bleeding, temporal trend, antithrombotic therapy, outcome

\section{INTRODUCTION}

The use of intensive antithrombotic therapies coupled with revascularization has been shown to reduce ischemic risk after an acute coronary syndrome (ACS) (1), but such strategies are performed at the expense of an increased risk of bleeding (2). Recent analyses showed that major bleeding events were equivalent or even more prognostic for death than spontaneous myocardial infarction $(3,4)$.

In particular, East Asian people have different risk profiles for both thrombophilia and bleeding and might be more susceptible to bleeding (5). For potent antithrombotic therapy, various reports have failed to show a net clinical benefit in East Asian patients in contrast to similar studies in Caucasian patients (6). There are several studies describing temporal changes in bleeding rates among Western populations during the past decades (7-9), but no such data are available in East Asian countries. Therefore, we used data from the CCC-ACS project (Improving Care for Cardiovascular Disease in China-Acute Coronary Syndrome) (10) to delineate the temporal trend of in-hospital major bleeding in relation to development of therapeutic strategies and outcomes from 2015 to 2019 among Chinese patients hospitalized for ACS.

\section{METHODS}

\section{Study Design and Population}

The CCC-ACS project (NCT02306616) is an ongoing nationwide registry and quality improvement project focusing on the quality of care for patients with ACS. The project was launched in November 2014 as a collaborative initiative of the American Heart Association and the Chinese Society of Cardiology. Details of the study design and methodology have been described elsewhere (10). In brief, the study included 159 tertiary hospitals and 82 secondary hospitals in China. In each hospital, the first 20 to 30 ACS patients of each month are consecutively recruited and identified based on principal discharge diagnosis. ACS was defined according to the guidelines published by the Chinese Society of Cardiology for the diagnosis and management of patients with ST-segment-elevation myocardial infarction (STEMI) and non-ST-segment elevation (NSTE)-ACS $(11,12)$. The Ethics Committee of Beijing Anzhen Hospital, Capital Medical University approved the study with a waiver for informed consent. The research was performed without patient or public involvement.

Overall, 113,650 inpatients with ACS from 241 hospitals were registered between November 2014 and December 2019. Of these, 113,567 patients were included in this study after excluding $83(0.07 \%)$ patients with missing in-hospital outcomes.

\section{Data Collection}

A standard web-based data collection platform (Oracle Clinical Remote Data Capture, Oracle) was used. Data elements included patients' characteristics, medical history, clinical presentation, diagnosis and risk evaluation, in-hospital management, discharge medications and in-hospital outcomes. Third-part clinical research associates performed regular quality audits to ensure that cases were reported consecutively rather than selectively.

\section{In-hospital Outcomes}

Major bleeding was defined as a composite of intracranial bleeding, retroperitoneal bleeding, a decline in hemoglobin levels $\geq 3 \mathrm{~g} / \mathrm{dL}$ during hospitalization, transfusion with overt bleeding, or bleeding requiring surgical intervention, and fatal bleeding. Major adverse cardiovascular events (MACE) included cardiac death, reinfarction, stent thrombosis, and ischemic stroke. Cardiac death was defined as death related to proximate cardiac causes, procedure-related complications, or any death unless an unequivocal non-cardiovascular cause could be established. All these outcomes were diagnosed by doctors during patients' hospitalization and recorded in medical records.

\section{Statistical Analysis}

Changes in patients' characteristics, in-hospital management and outcomes were evaluated annually (from 2015 to 2019). The number of patients in 2014 was small and thus added to the number of patients in 2015. For in-hospital major bleeding, patients were also stratified by sex, age, type of ACS [STEMI, non ST segment elevation myocardial infarction (NSTEMI), unstable angina pectoris (UAP)], diabetes mellitus, hypertension, eGFR at admission, Killip class, percutaneous coronary intervention (PCI), type of $\mathrm{P}_{2} \mathrm{Y}_{12}$ inhibitor, parenteral anticoagulation therapy, and GPIIb/IIIa inhibitor use during hospitalization. Continuous variables were shown as mean [standard deviation (SD)] unless otherwise indicated. Categorical variables were presented as the number (percentage). KruskalWallis test was used to examine the trend of major bleeding and ischemic outcomes over time.

To assess the effect of time on major bleeding, assuming a linear association for each 1-year time-block and outcome, a logistic regression model was fitted. These models explored the association for moving forward 1 year, with stepwise adjustments as follows: (1) crude; (2) age and gender; (3) baseline characteristics (diabetes mellitus, hypertension, eGFR < $60 \mathrm{~min}$ per $1.73 \mathrm{~m}^{2}$, STEMI, and Killip class); (4) inhospital antithrombotic treatments (GPIIb/IIIa inhibitor use, anticoagulant treatment, and ticagrelor use); and (5) invasive treatments [PCI (for STEMI, primary PCI), transradial access]. Standardization of rate of bleeding was performed using logistic regression models to account for the effect of differences in previously described patient characteristics and treatments throughout the observation period.

For variables with a missing rate of $<15 \%$, we imputed missing values using the sequential regression multiple imputation method implemented by IVEware software version 0.2 (Survey 
TABLE 1 | Baseline characteristics.

\begin{tabular}{|c|c|c|c|c|c|c|}
\hline & $\begin{array}{c}2015 \\
(n=29,957)\end{array}$ & $\begin{array}{c}2016 \\
(n=25,028)\end{array}$ & $\begin{array}{c}2017 \\
(n=18,979)\end{array}$ & $\begin{array}{c}2018 \\
(n=19,595)\end{array}$ & $\begin{array}{c}2019 \\
(n=20,008)\end{array}$ & $\begin{array}{c}2015-2019 \\
(n=113,567)\end{array}$ \\
\hline Age, y & $62.8 \pm 12.5$ & $62.9 \pm 12.4$ & $63.5 \pm 12.4$ & $64.0 \pm 12.4$ & $64.4 \pm 12.3$ & $63.4 \pm 12.4$ \\
\hline Hypertension & $19,446(64.9)$ & $16,372(65.4)$ & 12,637 (66.6) & 13,378 (68.3) & $13,779(68.9)$ & $75,612(67.2)$ \\
\hline Diabetes & $13,090(43.7)$ & $11,101(44.4)$ & $8,829(46.5)$ & $9,156(46.7)$ & $9,158(45.8)$ & $51,334(45.2)$ \\
\hline Previous PCl & $2,267(7.6)$ & $1,922(7.7)$ & $1,591(8.4)$ & $1,762(9.0)$ & 2,112 (10.6) & 9,654 (8.5) \\
\hline Previous CABG & $154(0.5)$ & $126(0.5)$ & $104(0.6)$ & $118(0.6)$ & $91(0.5)$ & $593(0.5)$ \\
\hline Previous atrial fibrillation & $798(2.7)$ & $538(2.2)$ & $424(2.2)$ & $486(2.5)$ & $460(2.3)$ & $2,706(2.4)$ \\
\hline Previous heart failure & $730(2.4)$ & $426(1.7)$ & $375(2.0)$ & $570(2.9)$ & 595 (3.0) & $2,696(2.4)$ \\
\hline Previous stroke & 3,017 (10.1) & 2,314 (9.3) & 1,666 (8.8) & $1,446(7.4)$ & $1,570(7.9)$ & $10,013(8.9)$ \\
\hline $\begin{array}{l}\text { Diastolic pressure, } \\
\mathrm{mmHg}\end{array}$ & $77.7 \pm 14.4$ & $78.2 \pm 14.4$ & $78.5 \pm 14.2$ & $79.3 \pm 14.6$ & $79.9 \pm 14.7$ & $79.9 \pm 14.7$ \\
\hline $\begin{array}{l}\text { Heart rate, beats per } \\
\text { min }\end{array}$ & $77.2 \pm 16.2$ & $77.6 \pm 16.3$ & $77.6 \pm 16.1$ & $77.9 \pm 16.3$ & $78.3 \pm 16.7$ & $78.3 \pm 16.7$ \\
\hline STEMI & 19,365 (64.6) & $15,429(61.7)$ & $10,910(57.5)$ & 10,476 (53.5) & $9,390(46.9)$ & $65,570(58.3)$ \\
\hline Cardiogenic shock & $1,257(6.5)$ & $974(6.3)$ & $675(6.2)$ & $599(5.7)$ & $601(6.4)$ & $4,106(6.3)$ \\
\hline Cardiac arrest & $687(2.3)$ & $439(1.8)$ & $195(1.0)$ & $125(0.6)$ & $111(0.6)$ & $1,557(1.4)$ \\
\hline Killip class & $20,213(67.5)$ & 17,096 (68.3) & $12,489(65.8)$ & $12,298(62.8)$ & 12,637 (63.2) & $74,733(65.8)$ \\
\hline \multicolumn{7}{|l|}{ Class I } \\
\hline Class II-III & 8,066 (26.9) & 6,614 (26.4) & 5,484 (28.9) & 6,230 (31.8) & 6,208 (31.0) & $32,602(28.7)$ \\
\hline Class IV & 1,678 (5.6) & $1,318(5.3)$ & $1,006(5.3)$ & $1,067(5.5)$ & 1,163 (5.8) & 6,232 (5.5) \\
\hline
\end{tabular}

CABG, coronary artery bypass grafting; eGFR, estimated glomerular filtration rate; MI, myocardial infarction; PCl, percutaneous coronary intervention.

Research Center, University of Michigan, Ann Arbor, MI, USA). Missing rates of variables and strategies for the management of missing data are presented in Supplementary Table 1. Statistical analyses were performed using SAS 9.4 (SAS Institute, Cary, NC, USA), SPSS 26.0 (IBM SPSS Inc., Armonk, NY) and Stata 14.0 (Stata, College Station, TX, USA). Two-tailed $p$-values of $<0.05$ were considered statistically significant.

\section{RESULTS}

Mean patient age of the whole cohort was $63.4 \pm 12.4$ years, and $27.1 \%$ were female. Most of the patients' characteristics showed only minor changes during the study period. There was a slight increase in the proportion of female patients and the prevalence of hypertension and previous PCI, and a decrease in the prevalence of previous stroke. The proportion of STEMI patients decreased steadily, associated with a temporal decline in patients with cardiac arrest (Table 1).

The use of antithrombotic therapies changed over time, with a fall in the use of anticoagulation therapy (77.5-60.6\%) during hospitalization among all ACS patients. There was a marked decrease in the use of GP IIb/IIIa inhibitors over time (34.1$19.6 \%)$. Use of PCI was high in all ACS patients with extremely high proportion of radial access throughout the whole study period. The proportion of primary PCI in STEMI patients also increased over time (Table 2).

\section{In-hospital Bleeding}

Overall, the rate of in-hospital major bleeding decreased from $6.3 \%$ in 2015 to $4.7 \%$ in 2019 (absolute change 1.6\%, relative change $25.4 \%$, unadjusted $\mathrm{OR}=0.74,95 \% \mathrm{CI}: 0.68-0.80$, and $P<0.001$; Tables 3, 4; Figure 1A). A similar trend was found in patients with overt bleeding and those without overt bleeding but with decline in hemoglobin levels $\geq 3 \mathrm{~g} / \mathrm{dL}$ (Table 4). The decrease of in-hospital bleeding occurred in parallel to the decreased use of GP IIb/IIIa inhibitors and anticoagulation therapy during hospitalization (Table 2; Figure 1B). Patients with NSTE-ACS (including NSTEMI and UAP) had a lower rate of bleeding than patients with STEMI, and similar trends were found in patients with NSTEMI and UAP (Table 4; 
TABLE 2 | In-hospital management*.

\begin{tabular}{|c|c|c|c|c|c|c|}
\hline & $\begin{array}{c}2015 \\
(n=29,957)\end{array}$ & $\begin{array}{c}2016 \\
(n=25,027)\end{array}$ & $\begin{array}{c}2017 \\
(n=18,979)\end{array}$ & $\begin{array}{c}2018 \\
(n=19,595)\end{array}$ & $\begin{array}{c}2019 \\
(n=20,008)\end{array}$ & $\begin{array}{c}2015-2019 \\
(n=113,567)\end{array}$ \\
\hline \multicolumn{7}{|l|}{ Antiplatelet therapy } \\
\hline None & $828(2.8)$ & 743 (3.0) & 636 (3.4) & $813(4.2)$ & $929(4.6)$ & 3,949 (3.5) \\
\hline Aspirin only & $499(1.7)$ & $572(2.3)$ & $606(3.2)$ & $984(5.0)$ & $1,184(5.9)$ & 3,845 (3.4) \\
\hline $\begin{array}{l}\mathrm{P}^{2} \mathrm{Y}_{12} \text { receptor } \\
\text { inhibitor only }\end{array}$ & $799(2.7)$ & $592(2.4)$ & $524(2.8)$ & $511(2.6)$ & $546(2.7)$ & 2,972 (2.6) \\
\hline DAPT & 27,831 (92.9) & 23,121 (92.4) & $17,213(90.7)$ & $17,287(88.2)$ & $17,349(86.7)$ & $102,801(91.4)$ \\
\hline Ticagrelor & 3,863 (13.5) & 4,788 (20.2) & 4,549 (25.7) & 6,408 (36.0) & 7,857 (43.9) & $27,465(24.4)$ \\
\hline $\begin{array}{l}\text { Glycoprotein Ilb/Illa } \\
\text { inhibitors }^{\dagger}\end{array}$ & $10,217(34.1)$ & $7,171(28.7)$ & $4,520(23.8)$ & 4,375 (22.3) & 3,925 (19.6) & $30,208(26.9)$ \\
\hline $\begin{array}{l}\text { Anticoagulation } \\
\text { therapy } \neq\end{array}$ & 23,207 (77.5) & $18,205(72.7)$ & 13,149 (69.3) & 12,477 (63.7) & $12,130(60.6)$ & 79,168 (70.4) \\
\hline $\mathrm{UFH}^{\ddagger}$ & $1,159(3.9)$ & 837 (3.3) & $657(3.5)$ & $483(2.5)$ & $932(4.7)$ & 4,068 (3.6) \\
\hline $\mathrm{LMWH}^{\ddagger}$ & 21,651 (72.3) & $16,831(67.3)$ & 12,109 (63.8) & $11,647(59.4)$ & $11,164(55.8)$ & $73,402(65.3)$ \\
\hline $\mathrm{UFH}$ or $\mathrm{LMWH}^{\ddagger}$ & 22,449 (74.9) & $17,385(69.5)$ & $12,511(65.9)$ & 11,935 (60.9) & 11,804 (59.0) & $76,084(67.0)$ \\
\hline Fondaparinux $\neq$ & $405(1.4)$ & $346(1.4)$ & $270(1.4)$ & $321(1.6)$ & $143(0.7)$ & $1,485(1.3)$ \\
\hline Others ${ }^{\ddagger}$ & $438(1.5)$ & $512(2.1)$ & $407(2.1)$ & $253(1.3)$ & $208(1.0)$ & $1,818(1.6)$ \\
\hline Warfarin & $186(0.6)$ & $168(0.7)$ & $142(0.8)$ & $170(0.9)$ & $126(0.6)$ & $792(0.7)$ \\
\hline$\beta$-blockers & $16,625(55.5)$ & $13,591(54.3)$ & 10,822 (57.0) & $11,455(58.5)$ & $10,978(54.9)$ & $63,471(55.9)$ \\
\hline ACEI or ARB & $14,439(48.2)$ & $11,755(47.0)$ & $9,178(48.4)$ & 9,502 (48.5) & $8,737(43.7)$ & $53,611(47.2)$ \\
\hline Statins & 28,094 (93.8) & 23,349 (93.3) & 17,658 (93.0) & 18,322 (93.5) & 18,665 (93.3) & $106,088(93.4)$ \\
\hline Coronary angiography & $21,378(71.4)$ & $18,981(75.8)$ & 14,893 (78.5) & 15,262 (77.9) & 14,761 (73.8) & $85,275(75.1)$ \\
\hline $\mathrm{PCl}$ & $19,372(64.7)$ & $18,142(72.5)$ & $13,641(71.9)$ & $13,473(68.8)$ & 12,917 (64.6) & $77,545(69.0)$ \\
\hline $\mathrm{DES}^{\S}$ & $16,556(85.5)$ & $15,197(83.8)$ & $11,687(85.7)$ & 11,934 (88.6) & $11,192(86.7)$ & $66,566(85.8)$ \\
\hline $\mathrm{BMS}^{\S}$ & $126(0.7)$ & $180(1.0)$ & $90(0.7)$ & $39(0.3)$ & $91(0.7)$ & $526(0.7)$ \\
\hline PTCA $^{\S}$ & 2,594 (13.4) & $2,575(14.2)$ & $1,768(13.0)$ & $1,476(11.0)$ & $1,570(12.2)$ & 9,983 (12.9) \\
\hline Others ${ }^{\S}$ & $96(0.5)$ & $190(1.1)$ & $96(0.7)$ & $24(0.2)$ & $64(0.5)$ & $470(0.6)$ \\
\hline $\begin{array}{l}\text { Primary PCl in STEMI } \\
\text { patients }\end{array}$ & 10,165 (52.5) & 8,537 (55.3) & 6,150 (56.4) & 6,337 (60.5) & 6,048 (64.4) & $37,237(56.8)$ \\
\hline Thrombolysis & 769 (2.6) & 447 (1.8) & 607 (3.2) & 837 (4.3) & 738 (3.7) & 3,398 (3.0) \\
\hline Transradial access & 19,792 (92.6) & $18,002(94.8)$ & $14,383(96.6)$ & $14,668(96.1)$ & $14,222(96.3)$ & $81,067(95.1)$ \\
\hline
\end{tabular}

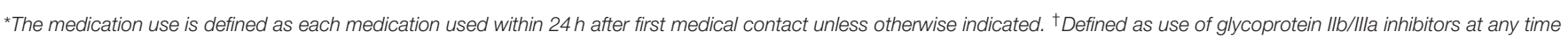
during hospitalization. ${ }^{\ddagger}$ Defined as use of anticoagulant during hospitalization but not during index procedure. ${ }^{\S}$ Denominator is the total number of PCI patients enrolled in each year.

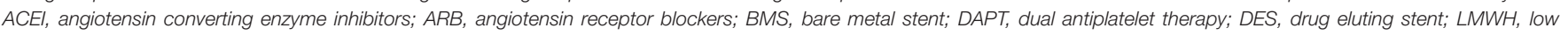

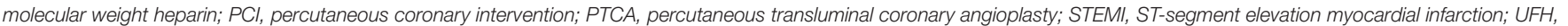
unfractionated heparin.

Figure 1A). The trend of bleeding was consistent across almost all subgroups including age, sex, eGFR, P2Y12 receptor inhibitor, and anticoagulation therapy (Table 4).

After adjustment for patient characteristics, medications, and interventions, the downward changes in bleeding were attenuated but remained for all ACS (Table 5). To evaluate the impact of changes in baseline characteristics and in-hospital management on outcomes, we did stepwise adjustments and analyzed the association between change in time-period and the risk of major bleeding. Each 1-year advancement was associated with reduced risk of bleeding in the crude analysis $(\mathrm{OR}=$ 0.91, 95\% CI: 0.89-0.93) and after adjustment for changes in demographics (OR $=0.91,95 \% \mathrm{CI}: 0.89-0.92)$. The OR increased to 0.93 (95\% CI: 0.91-0.94) after adjusting for baseline characteristics and further increased to 0.95 (95\% CI: $0.93-$ 0.97) after adjusting for antithrombotic treatments but did not change after adjusting for invasive treatment $(\mathrm{OR}=0.95,95 \% \mathrm{CI}$ :
0.93-0.97; Figure 2). For patients with NSTE-ACS, only changes in antithrombotic therapy explained the time related reduction in bleeding. However, for patients with STEMI, the association was attenuated after adjusting for changes in antithrombotic therapy and was no longer significant after further adjusting for invasive treatments (mainly primary PCI; Figure 2).

\section{Ischemic Outcomes}

The annual rate of reinfarction, stent thrombosis, and ischemic stroke remains relatively low and constant. The incidence of MACE decreased steadily with an absolute decrease of $1.5 \%$ (from 2.9 to $1.4 \%$ ), mainly driven by a decrease of cardiac death (from 2.2 to $0.8 \%$; Table 3; Figure 1C). The rate of all-cause death also decreased over time (from 2.3 to 1.4\%; Table 3). When combining ischemic and bleeding events together, a significant decrease in total events was also observed (Figure 1D). 
TABLE 3 | In-hospital outcomes in all ACS patients.

\begin{tabular}{|c|c|c|c|c|c|c|}
\hline & $\begin{array}{c}2015 \\
(n=29,957)\end{array}$ & $\begin{array}{c}2016 \\
(n=25,027)\end{array}$ & $\begin{array}{c}2017 \\
(n=18,979)\end{array}$ & $\begin{array}{c}2018 \\
(n=19,595)\end{array}$ & $\begin{array}{c}2019 \\
(n=20,008)\end{array}$ & $P$ for trend \\
\hline Major bleeding & 1,896 (6.3) & $1,521(6.1)$ & 994 (5.2) & $865(4.4)$ & $948(4.7)$ & $<0.001$ \\
\hline MACE & $859(2.9)$ & $528(2.1)$ & $318(1.7)$ & $326(1.7)$ & $288(1.4)$ & $<0.001$ \\
\hline Cardiac death & $650(2.2)$ & $383(1.5)$ & $212(1.1)$ & $217(1.1)$ & $167(0.8)$ & $<0.001$ \\
\hline Reinfarction & $124(0.4)$ & $75(0.3)$ & $72(0.4)$ & $86(0.4)$ & $79(0.4)$ & 0.14 \\
\hline Stent thrombosis & $65(0.2)$ & $43(0.2)$ & $15(0.1)$ & $18(0.1)$ & $15(0.1)$ & $<0.0001$ \\
\hline Ischemic stroke & $87(0.3)$ & $60(0.2)$ & $39(0.2)$ & $32(0.2)$ & $49(0.2)$ & 0.06 \\
\hline All-cause death & 689 (2.3) & $412(1.7)$ & 249 (1.3) & $272(1.4)$ & $278(1.4)$ & $<0.001$ \\
\hline
\end{tabular}

ACS, acute coronary syndrome; MACE, major adverse cardiovascular events.

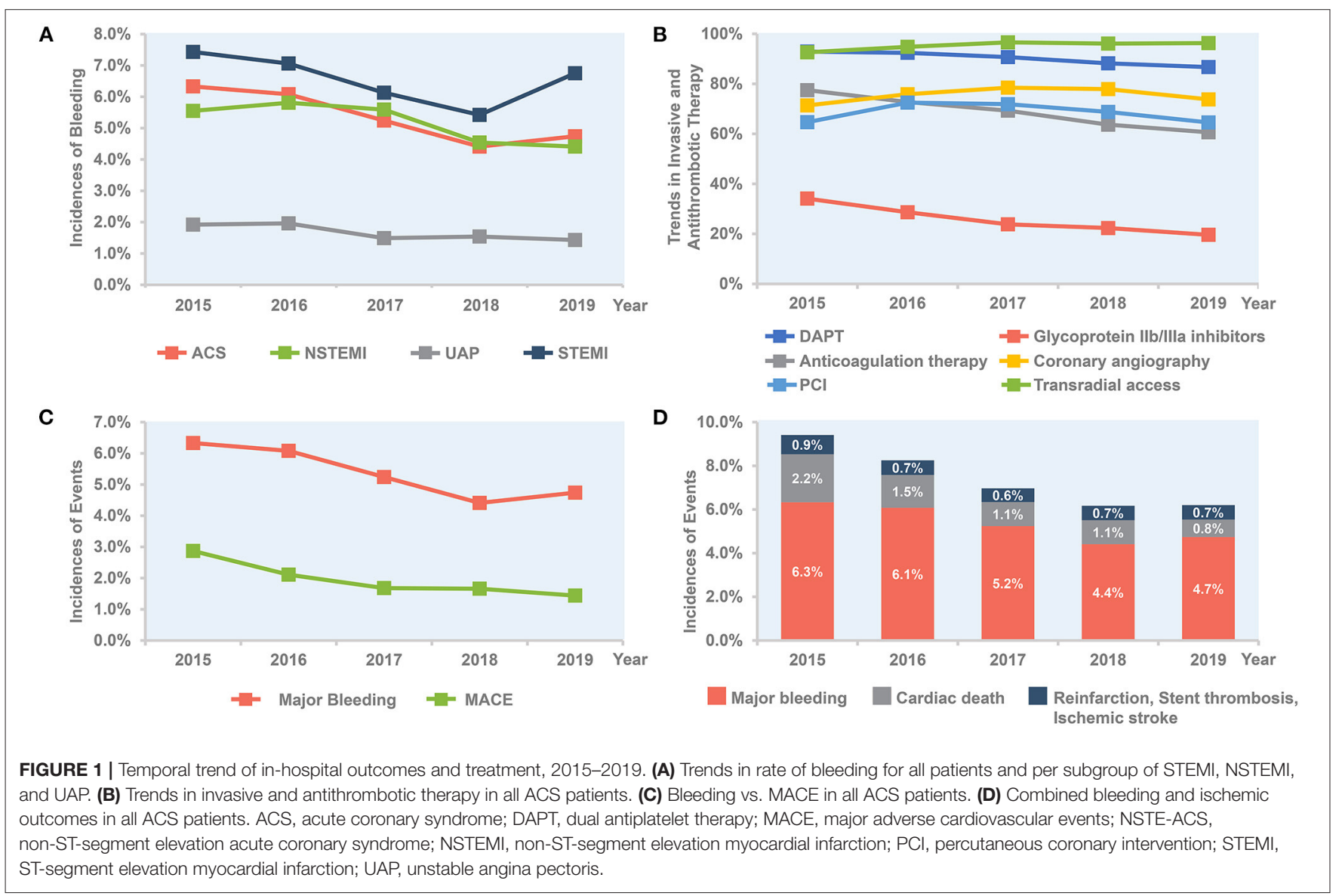

\section{DISCUSSION}

The present analysis from the large CCC-ACS registry showed an absolute $1.6 \%$ decrease in major bleeding in relation to more evidence-based use of antithrombotic therapies in patients hospitalized for ACS in China. Simultaneously, the incidence of ischemic events including cardiac death decreased steadily during the study period. To the best of our knowledge, this is the first report to delineate the temporal changes of bleeding events in East Asian countries with a higher risk profile for bleeding.
The study shows a continuous reduction in bleeding from 2015 to 2019, which is accompanied by a decrease in use of GP IIb/IIIa inhibitors and parenteral anticoagulation therapy during hospitalization. These findings are in line with recent studies from the SWEDEHEART registry (7) and UK database (8) showing a decline in in-hospital bleeding associated with less use of GP IIb/IIIa inhibitors. Specifically, the use of GP IIb/IIIa inhibitors was still as high as 20\% in 2019 compared with $4.7 \%$ in 2017/2018 in the SWEDEHEART registry (7). Currently, GP IIb/IIIa inhibitors are recommended on a "provisional" basis with decreased TIMI flow or new thrombus. Nevertheless, in 
TABLE 4 | Trends in rate of bleeding by patients' characteristics and in-hospital management.

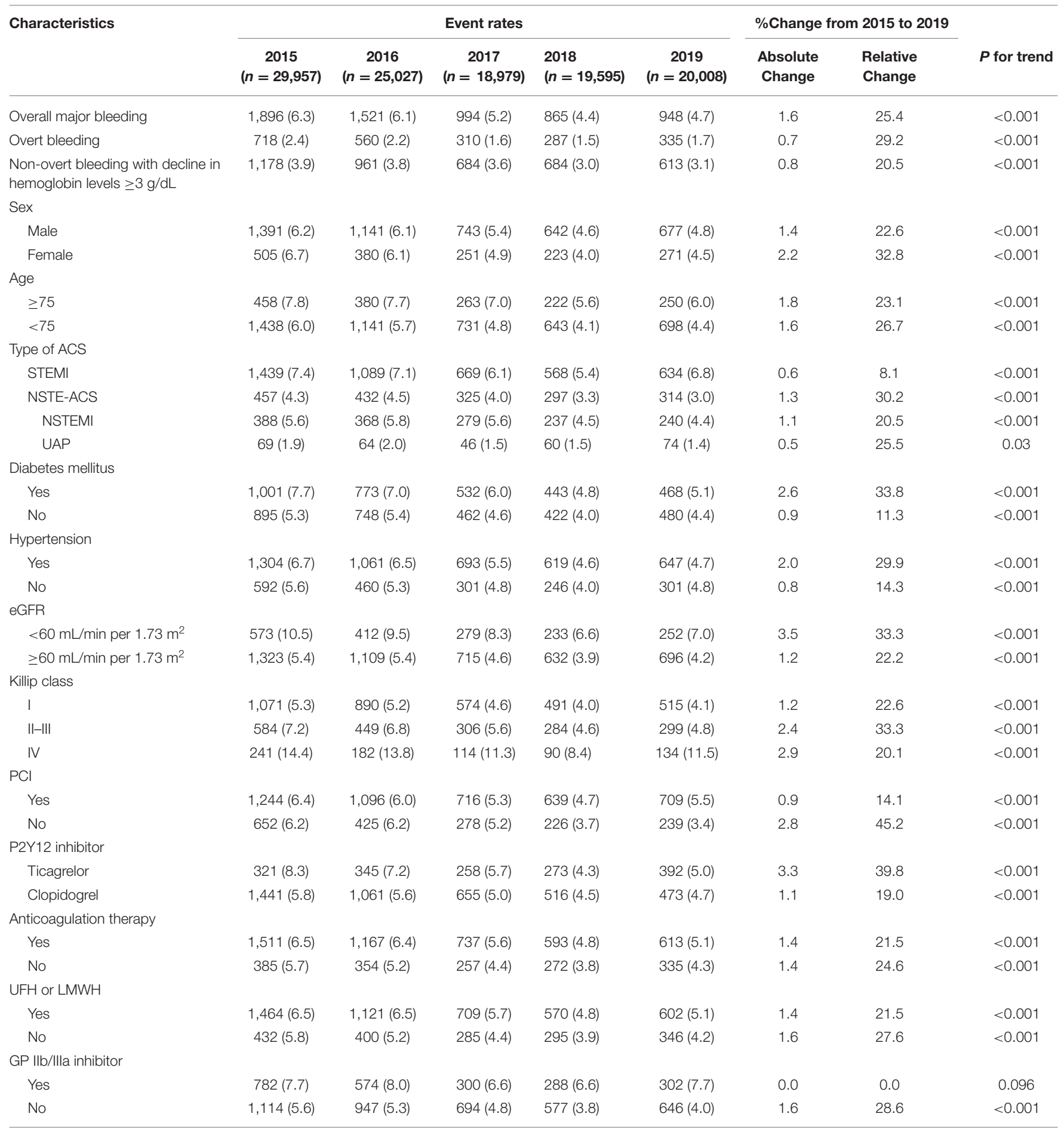

ACS, acute coronary syndrome; eGFR, estimated glomerular filtration rate; LMWH, low molecular weight heparin; NSTE-ACS, non-ST-segment elevation acute coronary syndrome; NSTEMI, non-ST-segment elevation myocardial infarction; PCI, percutaneous coronary intervention; STEMI, ST-segment elevation myocardial infarction; UAP, unstable angina pectoris; UFH, unfractionated heparin.

many secondary hospitals in China, GP IIb/IIIa inhibitors were almost routinely used during and/or after PCI in STEMI or NSTEMI patients, which actually have no robust evidence but may increase bleeding. Furthermore, anticoagulation therapy (especially the use of LMWH) was regarded as a default therapy following ACS or PCI among many centers in China. However, this strategy was not associated with a lower risk of all-cause death or myocardial infarction but significantly increased risk of major bleeding in the era of PCI and DAPT (13). The decrease from 77.5 to $60.6 \%$ in anticoagulation therapy might 
TABLE 5 | Standardized rate of bleeding in all ACS patients.

\begin{tabular}{|c|c|c|c|c|c|}
\hline & $\begin{array}{c}2015 \\
(n=29,957)\end{array}$ & $\begin{array}{c}2016 \\
(n=25,027)\end{array}$ & $\begin{array}{c}2017 \\
(n=18,979)\end{array}$ & $\begin{array}{c}2018 \\
(n=19,595)\end{array}$ & $\begin{array}{c}2019 \\
(n=20,008)\end{array}$ \\
\hline Model $1^{\star}$ & $6.4(6.1-6.7)$ & $6.1(5.8-6.4)$ & $5.2(4.9-5.6)$ & $4.4(4.1-4.7)$ & $4.7(4.4-5.0)$ \\
\hline Model $2^{\dagger}$ & $6.1(5.9-6.4)$ & $6.0(5.7-6.3)$ & $5.2(4.9-5.6)$ & $4.5(4.2-4.8)$ & $5.0(4.7-5.3)$ \\
\hline Model $3^{\ddagger}$ & $5.8(5.6-6.1)$ & $6.0(5.7-6.2)$ & $5.4(5.0-5.7)$ & $4.6(4.3-4.9)$ & $5.2(4.9-5.5)$ \\
\hline
\end{tabular}

${ }^{*}$ Adjusted for age and gender.

${ }^{\dagger}$ Adjusted for age, gender, and baseline characteristics (diabetes mellitus, hypertension eGFR<60 min per 1.73 m, STEMI, and Killip class).

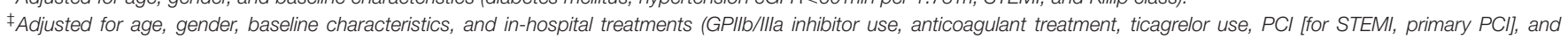
transradial access).

ACS, acute coronary syndrome; eGFR, estimated glomerular filtration rate; PCI, percutaneous coronary intervention; STEMI, ST-segment elevation myocardial infarction.

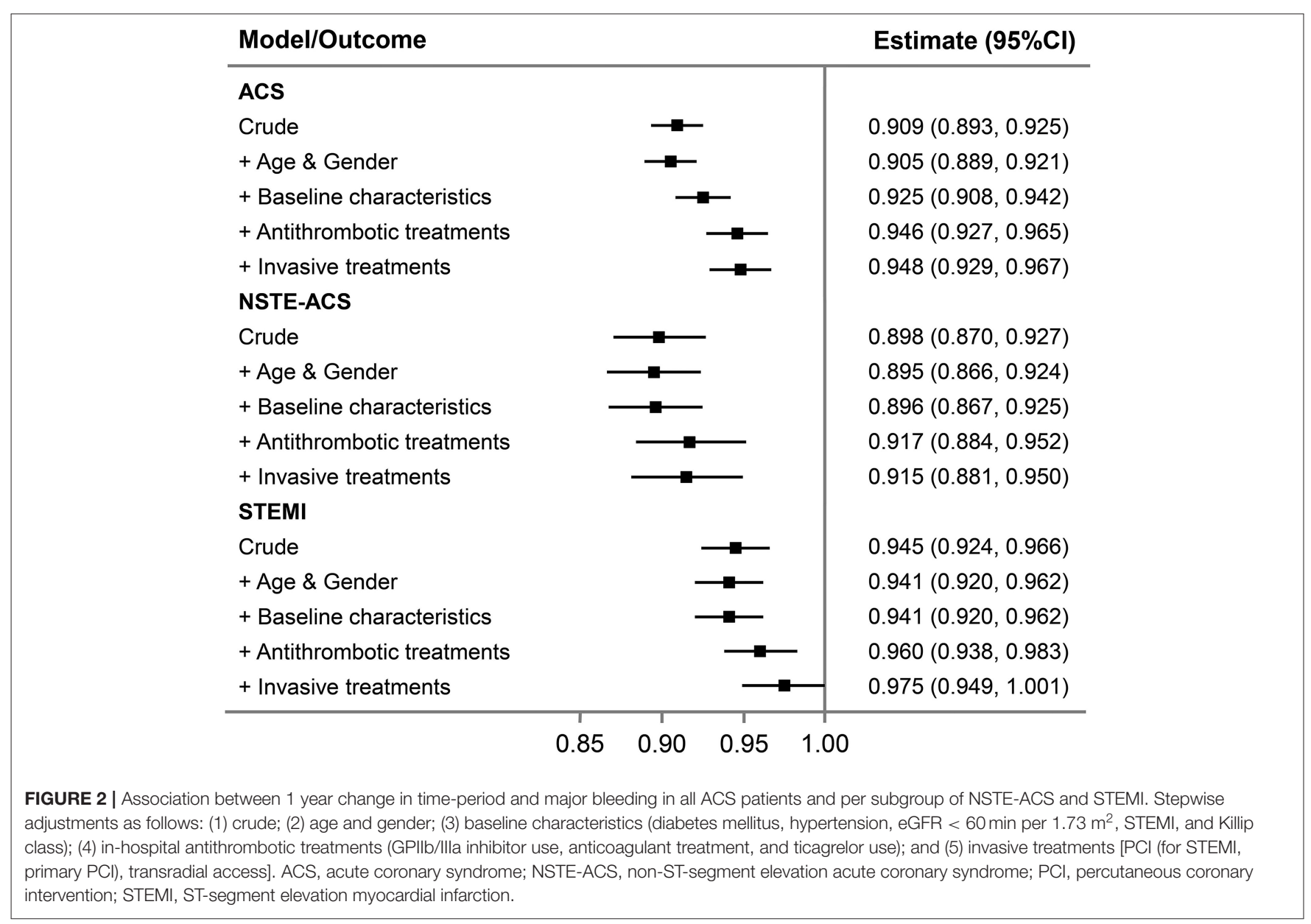

also have driven the decline in bleeding events. These findings were supported by stepwise adjustments for antithrombotic therapies, which showed attenuated association of time-period and bleeding trend.

In contrast to prior reports $(7,8,14)$, our study was performed on the background of high rates of radial access (95.1\% from 2015 to 2019) with no significant change over the past 5 years. This strategy is intuitively a bleeding reduction strategy as recommended by guidelines $(15,16)$. Recent study of Japanese population also showed low risk of bleeding complications by use of transradial approach or vascular closure device (17). However, data on vascular closure device was not collected in the CCC-ACS registry. Due to high rate of radial access in our study, vascular access strategies were minimally associated with these observed reductions in bleeding rates over time. The use of new-generation DES featuring much lower strut thickness and biodegradable polymers or polymer- free designs allows the shortening of DAPT (18-20). Also, use of intravascular imaging to optimize the PCI procedure and achieve more complete endothelialization is important in patients at high risk of bleeding who might need a short duration of DAPT (21). Additional strategies include the use of proton pump inhibitors to reduce risk of 
gastrointestinal bleeding who need antithrombotic medications $(22,23)$. Although not collected in this project, these factors should be further addressed. Finally, there was a steep rise in the rate of bleeding in STEMI patients from 2018 to 2019. We noted that the proportion of cardiogenic shock in STEMI patients showed a decrease from 2015 to 2018 but a sharp rise from 2018 to 2019 , which might at least partially explain this peculiar change. Other potential confounding factors may also take effects and should be further investigated.

In October 2016, the Chinese College of Cardiovascular Physicians Working Group on Thrombosis proposed a multidisciplinary expert consensus on the prevention and management of bleeding in patients with ACS receiving antithrombotic agents (24). The consensus proposed a multidisciplinary bleeding prevention and treatment framework with the cardiologist as the leader and several bleeding avoidance therapies including bleeding risk evaluation, tailored antithrombotic regimen, avoiding unnecessary in-hospital anticoagulation, crossover of anticoagulants, and use of GP IIb/IIIa inhibitors, and early use of PPI, which may partly explain the decline in bleeding rate after 2016.

The higher bleeding rate in our study compared with other reports maybe be partly attributed to higher proportion of STEMI patients and more use of GP IIb/IIIa inhibitors ( $\approx 20$ vs. $<5 \%$ in other registries) $(7,8)$ and anticoagulants. Actually, various studies have reported that East Asian people might be less prone to thrombosis and more prone to bleeding, especially in the context of potent antithrombotic therapy (25). In spite of this, our registry showed a faster and more substantial decrease in bleeding (2015-2019, from 6.3 to $4.7 \%$, absolute change 1.6\%) compared with the SWEDEHEART registry (2006/2007 to 2015/2016, from 2.0 to $1.3 \%$, absolute change $0.7 \%$ ).

On the other hand, the absolute reduction of MACE including cardiac death is also intriguing. In detail, the ischemic events including reinfarction, stent thrombosis, and ischemic stroke did not change a lot across the study. Although precisely causal link should be clearly established, we speculate the reduced rate of cardiac death might be in part owing to the decline of bleeding, as the latter may cause premature discontinuation of antithrombotic medications, reduced myocardial oxygen delivery due to hypotension and anemia, and blood transfusion (26). In the present study, we did not observe plateauing in bleeding rates, indicating that there is room for further lowering of event rates by further expansion of recommended therapies, which have not been fully exploited [e.g., high rate of GP IIb/IIIa inhibitors and anticoagulants use in 2019 compared to other registries $(7,8)]$.

\section{Limitations}

First, bleeding events were self-reported and not adjudicated, possibly leading to underreporting. Second, the standardized bleeding definitions which were usually used in clinical trials were not available in our registry, although the definitions remained unchanged during the study period. However, our bleeding definition is similar to BARC 3 or 5 bleeding. Third, given the observational design of this study, the cause-effect relationship should not be established between change in antithrombotic therapy and temporal reduction in bleeding. Fourth, the information of IABP, which might be associated with bleeding, was only available from 2017 to 2019 and was not presented. Fifth, whether bleeding was access site related or not was not collected in this registry. Sixth, out of hospital events were also not reported. Seventh, data regarding the use of bivalirudin and new oral anticoagulants, which have been related to lower risk of bleeding, were not collected due to their infrequent use. Finally, the effects of timing, dosing, and crossover of anticoagulants and antiplatelets could not be assessed based on this registry.

\section{CONCLUSIONS}

The present study shows a temporal reduction in in-hospital bleeding among Chinese ACS patients during the last 5 years. The changes in bleeding are largely associated with the decreased use of GP IIb/IIIa inhibitors and anticoagulation therapy. Given a higher risk profile for bleeding in the Chinese population, a more consistent and systematic application of evidence-based therapies and promotion of new treatment concepts are needed to further reduce bleeding risk and improve overall outcomes.

\section{DATA AVAILABILITY STATEMENT}

The datasets analyzed during the current study are not publicly available because of intellectual property rights, but are available from the corresponding author on reasonable request.

\section{ETHICS STATEMENT}

The studies involving human participants were reviewed and approved by Ethics Committee of Beijing Anzhen Hospital, Capital Medical University. Written informed consent for participation was not required for this study in accordance with the national legislation and the institutional requirements.

\section{AUTHOR CONTRIBUTIONS}

XW, GZ, SN, and YaH: study concept and design. XW, GZ, MZ, YoH, JuL, JiL, DZ, SN, and YaH: acquisition, analysis, or interpretation of data. XW and GZ: drafting of the manuscript. XW, GZ, and SN had full access to all the data in the study and take responsibility for the integrity of the data and the accuracy of the data analysis. All authors critical revision of the manuscript for important intellectual content, read, and approved the final manuscript.

\section{FUNDING}

This work was supported by a collaborative program of the American Heart Association (AHA) and the Chinese Society of Cardiology. The AHA was funded by Pfizer for the quality improvement initiative through an independent grant for learning and change. 


\section{ACKNOWLEDGMENTS}

The authors thank all participating hospitals for their contributions to the CCC-ACS project (Improving Care for Cardiovascular Disease in China-Acute Coronary Syndrome).

\section{REFERENCES}

1. Szummer K, Wallentin L, Lindhagen L, Alfredsson J, Erlinge D, Held $\mathrm{C}$, et al. Relations between implementation of new treatments and improved outcomes in patients with non-ST-elevation myocardial infarction during the last 20 years: experiences from SWEDEHEART registry 1995 to 2014. Eur Heart J. (2018) 39:3766-76. doi: 10.1093/eurheartj/ ehy554

2. Steg PG, Huber K, Andreotti F, Arnesen H, Atar D, Badimon L, et al. Bleeding in acute coronary syndromes and percutaneous coronary interventions: position paper by the Working Group on Thrombosis of the European Society of Cardiology. Eur Heart J. (2011) 32:1854-64. doi: 10.1093/eurheartj/ ehr204

3. Marquis-Gravel G, Dalgaard F, Jones AD, Lokhnygina Y, James SK, Harrington RA, et al. Post-discharge bleeding and mortality following acute coronary syndromes with or without PCI. J Am Coll Cardiol. (2020) 76:16271. doi: 10.1016/j.jacc.2020.05.031

4. Piccolo R, Oliva A, Avvedimento M, Franzone A, Windecker S, Valgimigli M, et al. Mortality after bleeding versus myocardial infarction in coronary artery disease: a systematic review and meta-analysis. EuroIntervention. (2021) 17:550-60. doi: 10.4244/EIJ-D-20-01197

5. Kang J, Park KW, Palmerini T, Stone GW, Lee MS, Colombo A, et al. Racial differences in ischaemia/bleeding risk trade-off during anti-platelet therapy: individual patient level landmark meta-analysis from seven RCTs. Thromb Haemost. (2019) 119:149-62. doi: 10.1055/s-0038-1676545

6. Park DW, Kwon O, Jang JS, Yun SC, Park H, Kang DY, et al. Clinically significant bleeding with ticagrelor versus clopidogrel in korean patients with acute coronary syndromes intended for invasive management: a randomized clinical trial. Circulation. (2019) 140:1865-77. doi: 10.1161/CIRCULATIONAHA.119.041766

7. Simonsson M, Wallentin L, Alfredsson J, Erlinge D, Hellstrom Angerud K, Hofmann R, et al. Temporal trends in bleeding events in acute myocardial infarction: insights from the SWEDEHEART registry. Eur Heart J. (2020) 41:833-43. doi: 10.1093/eurheartj/ehz593

8. Olier I, Carr M, Curzen N, Ludman P, Baumbach A, Kinnaird T, et al. Changes in periprocedural bleeding complications following percutaneous coronary intervention in the United Kingdom between 2006 and 2013 (from the British Cardiovascular Interventional Society). Am J Cardiol. (2018) 122:952-60. doi: 10.1016/j.amjcard.2018.06.016

9. Subherwal S, Peterson ED, Dai D, Thomas L, Messenger JC, Xian Y,et al. Temporal trends in and factors associated with bleeding complications among patients undergoing percutaneous coronary intervention: a report from the National Cardiovascular Data CathPCI Registry. J Am Coll Cardiol. (2012) 59:1861-9. doi: 10.1016/j.jacc.2011.12.045

10. Hao Y, Liu J, Liu J, Smith SC Jr, Huo Y, Fonarow GC, et al. Rationale and design of the Improving Care for Cardiovascular Disease in China (CCC) project: a national effort to prompt quality enhancement for acute coronary syndrome. Am Heart J. (2016) 179:107-15. doi: 10.1016/j.ahj.2016.06.005

11. China Society of Cardiology of Chinese Medical Association. Guideline for diagnosis and treatment of patients with STelevation myocardial infarction. Chin J Cardiol. (2010) 38:675-90. doi: 10.3760/cma.j.issn.0253-3758.2010.08.002

12. China Society of Cardiology of Chinese Medical Association. Guidelines for the management of acute coronary syndromes in patients presenting without persistent ST-segment elevation 2012. Chin J Cardiol. (2012) 40:353-67. doi: 10.3760/cma.j.issn.0253-3758.2012.05.001

13. Chen JY, He PC, Liu YH, Wei XB, Jiang L, Guo W, et al. Association of parenteral anticoagulation therapy with outcomes in Chinese

\section{SUPPLEMENTARY MATERIAL}

The Supplementary Material for this article can be found online at: https://www.frontiersin.org/articles/10.3389/fcvm. 2021.769165/full\#supplementary-material

patients undergoing percutaneous coronary intervention for non-STsegment elevation acute coronary syndrome. JAMA Intern Med. (2019) 179:186-94. doi: 10.1001/jamainternmed.2018.5953

14. Elbarouni B, Elmanfud O, Yan RT, Fox KA, Kornder JM, Rose B,et al. Temporal trend of in-hospital major bleeding among patients with non ST-elevation acute coronary syndromes. Am Heart J. (2010) 160:4207. doi: 10.1016/j.ahj.2010.05.036

15. Neumann FJ, Sousa-Uva M, Ahlsson A, Alfonso F, Banning AP, Benedetto U, et al. 2018 ESC/EACTS Guidelines on myocardial revascularization. EuroIntervention. (2019) 14:1435-534. doi: 10.4244/ EIJY19M01_01

16. Capodanno D, Bhatt DL, Gibson CM, James S, Kimura T, Mehran R, et al. Bleeding avoidance strategies in percutaneous coronary intervention. Nat Rev Cardiol. (2021) doi: 10.1038/s41569-021-00598-1. [Epub ahead of print].

17. Sawano M, Spertus JA, Masoudi FA, Rumsfeld JS, Numasawa Y, Inohara T, et al. Bleeding avoidance strategies and percutaneous coronary intervention outcomes: a 10-year observation from a Japanese Multicenter Registry. Am Heart J. (2021) 235:113-24. doi: 10.1016/j.ahj.2021.01.010

18. Mehran R, Cao D, Angiolillo DJ, Bangalore S, Bhatt DL, Ge J, et al. 3- or 1-month DAPT in patients at high bleeding risk undergoing everolimus-eluting stent implantation. JACC Cardiovasc Interv. (2021) 14:1870-83. doi: 10.1016/j.jcin.2021.07.016

19. Kirtane AJ, Stoler R, Feldman R, Neumann FJ, Boutis L, Tahirkheli N, et al. Primary results of the EVOLVE Short DAPT study: evaluation of 3-month dual antiplatelet therapy in high bleeding risk patients treated with a bioabsorbable polymer-coated Everolimus-eluting stent. Circ Cardiovasc Interv. (2021) 14:e010144. doi: 10.1161/CIRCINTERVENTIONS.120. 010144

20. Kandzari DE, Kirtane AJ, Windecker S, Latib A, Kedhi E, Mehran R, et al. One-month dual antiplatelet therapy following percutaneous coronary intervention with zotarolimus-eluting stents in high-bleeding-risk patients. Circ Cardiovasc Interv. (2020) 13:e009565. doi: 10.1161/CIRCINTERVENTIONS.120.009565

21. Buccheri S, Franchina G, Romano S, Puglisi S, Venuti G, D’Arrigo P, et al. Clinical outcomes following intravascular imaging-guided versus coronary angiography-guided percutaneous coronary intervention with stent implantation: a systematic review and Bayesian network meta-analysis of 31 studies and 17,882 patients. JACC Cardiovasc Interv. (2017) 10:248898. doi: 10.1016/j.jcin.2017.08.051

22. Capodanno D, Alfonso F, Levine GN, Valgimigli M, Angiolillo DJ. ACC/AHA versus ESC guidelines on dual antiplatelet therapy: JACC guideline comparison. J Am Coll Cardiol. (2018) 72:2915-31. doi: 10.1016/j.jacc.2018.09.057

23. Bhatt DL, Cryer BL, Contant CF, Cohen M, Lanas A, Schnitzer TJ, et al. Clopidogrel with or without omeprazole in coronary artery disease. $N$ Engl J Med. (2010) 363:1909-17. doi: 10.1056/NEJMoa1007964

24. Chinese College of Cardiovascular P, Chinese College of Cardiovascular Physicians Working Group on $\mathrm{T}$, Chinese Society of Digestive E. Multidisciplinary expert consensus on the prevention and management of bleeding in patients with acute coronary syndrome receiving antithrombotic agents. Zhonghua Nei $\mathrm{Ke} \mathrm{Za} Z \mathrm{Zhi}$. (2016) 55:813-24. doi: 10.3760/cma.j.issn.0578-1426.2016.10.021

25. Huo Y, Jeong Y-H, Gong Y, Wang D, He B, Chen J, et al. 2018 update of expert consensus statement on antiplatelet therapy in East Asian patients with ACS or undergoing PCI. Sci Bull. (2019) 64:166-79. doi: 10.1016/j.scib.2018. 12.020

26. Genereux P, Giustino G, Witzenbichler B, Weisz G, Stuckey TD, Rinaldi $\mathrm{MJ}$, et al. Incidence, predictors, and impact of post-discharge bleeding after 
percutaneous coronary intervention. J Am Coll Cardiol. (2015) 66:103645. doi: 10.1016/j.jacc.2015.06.1323

Conflict of Interest: GF consulted for Amgen, AstraZeneca, Bayer, Janssen, and Novartis and served on the AHA's Quality Oversight Committee. CM received honoraria from Bristol-Myers Squibb (BMS), Pfizer, Johnson \& Johnson, Boehringer-Ingelheim (BI), Bayer and AstraZeneca for giving lectures. SN received research grants from the institution from Boston Scientific, Abbott, Jiangsu Hengrui Pharmaceuticals, China Resources Sanjiu Medical \& Pharmaceuticals, East China Pharmaceuticals.

The remaining authors declare that the research was conducted in the absence of any commercial or financial relationships that could be construed as a potential conflict of interest.
Publisher's Note: All claims expressed in this article are solely those of the authors and do not necessarily represent those of their affiliated organizations, or those of the publisher, the editors and the reviewers. Any product that may be evaluated in this article, or claim that may be made by its manufacturer, is not guaranteed or endorsed by the publisher.

Copyright (c) 2021 Wang, Zhao, Zhou, Ma, Ge, Huo, Smith, Fonarow, Hao, Liu, Morgan, Gong, Yan, Liu, Zhao, Han and Nie. This is an open-access article distributed under the terms of the Creative Commons Attribution License (CC BY). The use, distribution or reproduction in other forums is permitted, provided the original author(s) and the copyright owner(s) are credited and that the original publication in this journal is cited, in accordance with accepted academic practice. No use, distribution or reproduction is permitted which does not comply with these terms. 